\title{
New Model Sequences from the White Dwarf Evolution Code
}

\author{
M. WOOD
}

Department of Physics and Space Sciences, Florida Institute of Technology, Melbourne, FL 92901-6988 USA

\begin{abstract}
Model sequences computed with the recently-published OPAL radiative opacities, Itoh et al. conductive opacities, and Itoh et al. neutrino rates are presented. Cooling times for DA model sequences are found to be independent of metallicity for $Z \leq 0.001$.
\end{abstract}

\subsection{Introduction}

In the past decade, many improvements in the constitutive physics relevant to white dwarf evolutionary models have been published. These include improved radiative opacities (Rogers \&. Iglesias 1992; Iglesias \& Rogers 1993), conductive opacities for pure (Itoh et al. 1993 and references therein) and mixed (Itoh \& Kohyama 1993) compositions, and updated neutrino rates (Itoh et al. 1992 and references therein). We have incorporated these results into our white dwarf evolution code (=WDEC; see Lamb \& Van Horn 1975 , and Wood 1990), and present here selected C-core DA model sequences computed with the updated code. Stellar masses for the sequences are $0.4,0.6$, and $0.8 M_{\odot}$ and surface layer masses are $\log q(H)=-6$ and $\log q(\mathrm{He})=-4$. To determine the effect of metallicity on the evolutionary timescale, we computed parallel sequences with $Z=0.000$ and 0.001 . 


\subsection{Opacities}

The radiative opacities used in WDEC in the past (Cox \& Stewart 1970) had an unrealistically-high metallicity of $Z=0.001\left(Z_{\text {obs }} \lesssim 10^{-5}\right)$ The new OPAL opacities span a wide range of metallicities and compositions, and therefore allow the inclusion of more plausible composition profiles in the models. The OPAL opacities only extend to a minimum temperature of $6000 \mathrm{~K}$, however, so for DA models WDEC references the pure-H opacities of Lenzuni et al. (1991) below this point.

Figure 1 shows the $\rho-T$ domain of interest, with the crosshatched regions showing the extents of the various opacity tables. Also included on this plot are three $0.6 M_{(\ominus)}$ models with effective temperatures of $173,000 \mathrm{~K}$, $12,300 \mathrm{~K}$, and $3780^{\circ} \mathrm{K}$. Note that for models cooler than $\sim 15,000 \mathrm{~K}$, the opacities for shells in the outer envelope must be extrapolated off the OPAL (and Itoh et al.) tables. The extrapolations are along an isotherm and are linear in the logarithms of density and opacity. Of hydrogen, helium, or carbon, it is the helium opacity which shows the greatest sensitivity to metallicity between $Z=0.000$ and 0.001 with $\Delta \kappa_{\max } \approx 5$ dex (but only for $T<20,000 \mathrm{~K}$ ); for hydrogen and carbon (and helium above $20,000 \mathrm{~K}$ ), we find $\Delta \kappa_{\max } \approx 0.5$ dex. As a result of these small differences, we expect to also find a small sensitivity to metallicity in the model ages, and this indeed is the case (see below).

Unfortunately, because no radiative or conductive opacities currently exist for this dual-extrapolation region, the absolute ages of the low-luminosity models are still uncertain (see also Mazzitelli's contribution in these proceedings). However, one of the positive outcomes of this meeting was a formal letter to $F$. Rogers from a few of us in the white dwarf modelling community requesting an extension of his tables to $\log R=+5$. This will essentially fill in the current opacity "hole," and should be a feasible extension, according to Rogers (private communication). We can anticipate that within a few months' time the ages of the cool white dwarf models will be on much firmer ground than is now the case.

The conductive opacities of $\mathrm{N}$. Itoh and collaborators were introduced a decade ago, and these and subsequent refinements have been included into WDEC. The low-density, boundary to these tables is approximately given by the condition $T \leq 0.1 T_{\mathrm{Fermi}}$. It would be most useful if these calculations could be extended to lower densities. 


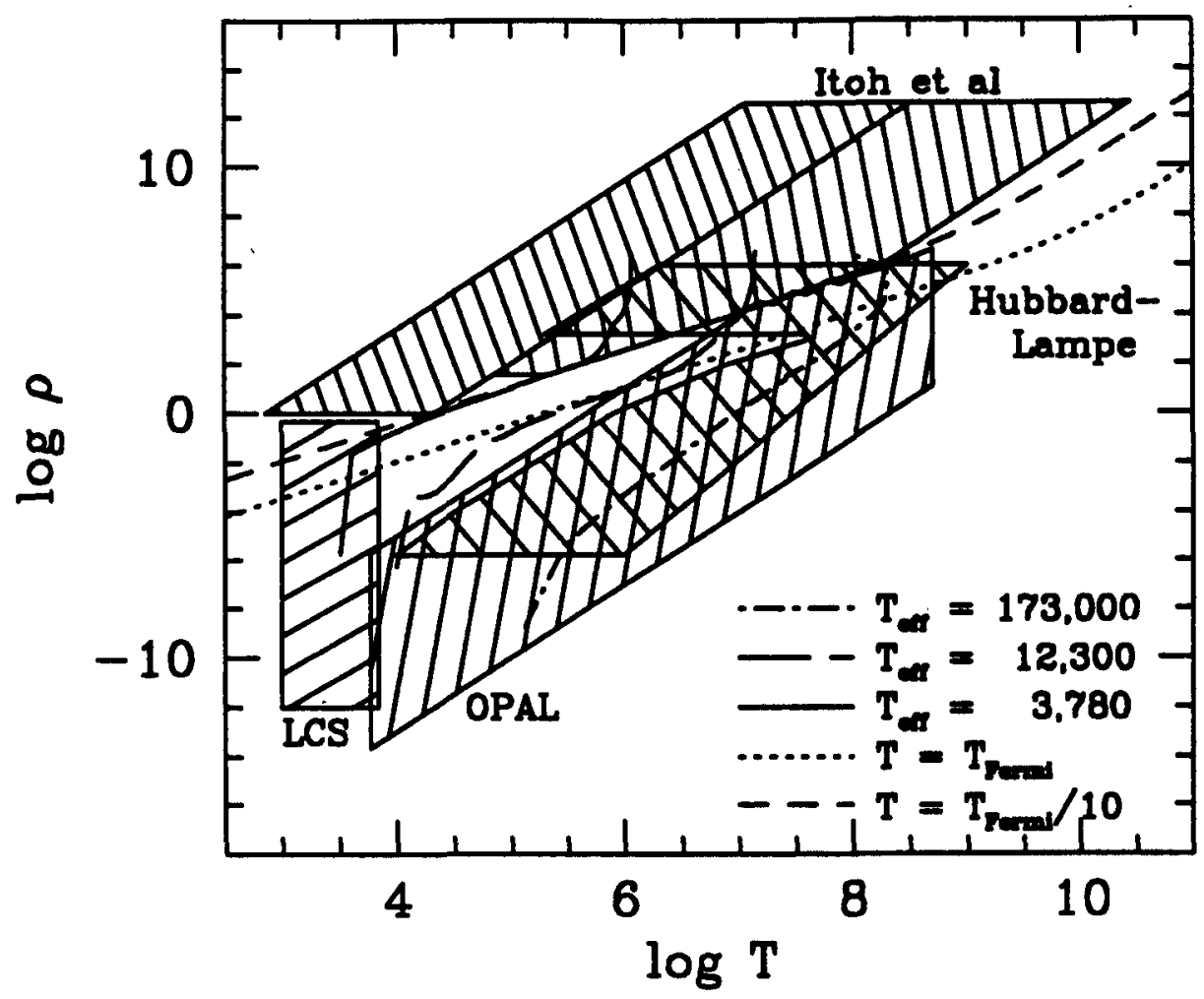

Fig. 42.1 Opacity tables' domains in $\rho-T$ plane. Also shown are the $\rho-T$ loci for models with the indicated effective temperatures.

\subsection{Neutrino Rates}

The neutrino rates of Itoh and collaborators are comparable to the rates of Beaudet et al. (1967) and De Zotti (1972). The largest difference between the old and new rates is for the bremsstrahlung process, which is roughly equivalent at high temperatures, but a factor of three or more weaker at low temperatures.

\subsection{Results}

In order to explore the importance of surface metallicity, I compnted computed parallel $0.4,0.6$, and $0.8 M_{\odot}$ C-core DA sequences with $Z=0.000$ and 0.001 . The evolutionary summary listings for the $Z=0.000$ sequences are given in Tables 1-3, sampled every 0.5 dex in $\log \left(L / L_{\odot}\right)$ before crystallization onset, and every 0.2 dex thereafter†. Comparing, we find that

t The listings of these and other sequences are available in electronic form. Flease send e-mail requests to wood(i)kepler.pss.fit.edu. 
Table 42.1. Evolutionary summary of a $Z=0.0000 .4 M_{\odot}$ model sequence

\begin{tabular}{ccccccccc}
\hline \hline $\log \left(L / L_{\odot}\right)$ & \multicolumn{2}{c}{ Age $(\mathrm{yr})$} & \multicolumn{1}{c}{$T_{\text {eff }}$} & $\log T_{c}$ & $\log \left(\rho_{c}\right)$ & $\log (R)$ & $\log \left(L_{\nu} / L_{\odot}\right)$ & $q_{\text {xtal }}$ \\
\hline 2.5 & 1.718 & $(2)$ & 113900 & 7.674 & 5.890 & 9.506 & -0.415 & 0.00 \\
2.0 & 5.790 & $(3)$ & 96064 & 7.677 & 5.894 & 9.404 & -0.392 & 0.00 \\
1.5 & 2.679 & $(4)$ & 79034 & 7.681 & 5.900 & 9.323 & -0.363 & 0.00 \\
1.0 & 1.133 & $(5)$ & 63668 & 7.690 & 5.908 & 9.261 & -0.324 & 0.00 \\
0.5 & 4.801 & $(5)$ & 50631 & 7.718 & 5.919 & 9.210 & -0.279 & 0.00 \\
0.0 & 1.944 & $(6)$ & 39962 & 7.791 & 5.937 & 9.166 & -0.286 & 0.00 \\
-0.5 & 6.405 & $(6)$ & 31311 & 7.796 & 5.965 & 9.128 & -0.533 & 0.00 \\
-1.0 & 1.771 & $(7)$ & 24307 & 7.709 & 5.993 & 9.098 & -1.072 & 0.00 \\
-1.5 & 5.009 & $(7)$ & 18703 & 7.581 & 6.014 & 9.075 & -1.928 & 0.00 \\
-2.0 & 1.473 & $(8)$ & 14294 & 7.410 & 6.029 & 9.059 & -3.263 & 0.00 \\
-2.5 & 3.757 & $(8)$ & 10870 & 7.218 & 6.039 & 9.047 & -4.979 & 0.00 \\
-3.0 & 8.646 & $(8)$ & 8249 & 7.008 & 6.045 & 9.036 & -6.502 & 0.00 \\
-3.5 & 1.821 & $(9)$ & 6244 & 6.783 & 6.049 & 9.028 & -8.357 & 0.00 \\
-4.0 & 4.180 & $(9)$ & 4736 & 6.465 & 6.052 & 9.018 & - & 0.00 \\
-4.2 & 5.403 & $(9)$ & 4225 & 6.353 & 6.053 & 9.017 & - & 0.02 \\
-4.3 & 6.351 & $(9)$ & 3990 & 6.294 & 6.054 & 9.017 & - & 0.22 \\
-4.4 & 7.501 & $(9)$ & 3769 & 6.231 & 6.054 & 9.017 & - & 0.46 \\
-4.5 & 8.637 & $(9)$ & 3559 & 6.173 & 6.054 & 9.016 & - & 0.64 \\
-4.6 & 9.707 & $(9)$ & 3361 & 6.117 & 6.054 & 9.016 & - & 0.77 \\
-4.7 & $1.078(10)$ & 3173 & 6.063 & 6.054 & 9.016 & - & 0.86 \\
-4.8 & $1.187(10)$ & 2996 & 6.009 & 6.055 & 9.016 & - & 0.91 \\
-5.0 & $1.412(10)$ & 2670 & 5.901 & 6.055 & 9.016 & - & 0.97 \\
-5.2 & $1.657(10)$ & 2380 & 5.792 & 6.055 & 9.016 & - & 0.99 \\
-5.3 & 1.790 & $(10)$ & 2247 & 5.734 & 6.055 & 9.016 & - & 1.00 \\
\hline \hline
\end{tabular}

the fractional age differences are always below $1 \%$ for the 0.4 and $0.6 M_{\odot}$ models, and $2 \%$ or below for the $0.8 M_{(\cdot)}$ models - i.e., the cooling times are relatively insensitive to metallicity for $Z \leq 0.001$. We note, however, that a 0.6 model sequence with a pure- $C$ composition is $20 \%$ older than the DA sequence at $\log \left(L / L_{\odot}\right)=-4.4$, and nearly a factor of 2 older at $\log \left(L / L_{\odot}\right)=-4.9$.

\subsection{Future Work}

Future work includes, first, publishing the current white dwarf model sequences so they are available in the literature; this is progressing. Italo Mazzitelli has kindly provided source code which implements the Canuto \& Mazzitelli $(1991,1992)$ convection theory, and this will be includer. We will also use the Lamb (1974) equation of state code to compute an interior EOS tables of $\mathrm{He}, \mathrm{Mg}$, and Ne so that we can compute more realistic low- 
Table 42.2. Evolutionary summary of a $Z=0.0000 .6 M_{\odot}$ model sequence

\begin{tabular}{ccccccccc}
\hline \hline $\log \left(L / L_{\odot}\right)$ & \multicolumn{2}{c}{ Age $(\mathrm{yr})$} & \multicolumn{1}{c}{$T_{\text {eff }}$} & $\log T_{c}$ & $\log \left(\rho_{c}\right)$ & $\log (R)$ & $\log \left(L_{\nu} / L_{\odot}\right)$ & $q_{\text {xtal }}$ \\
\hline 3.0 & 2.163 & $(3)$ & 162459 & 8.012 & 6.301 & 9.448 & 1.503 & 0.00 \\
2.5 & 1.065 & $(4)$ & 140649 & 8.016 & 6.311 & 9.323 & 1.542 & 0.00 \\
2.0 & 5.024 & $(4)$ & 117608 & 8.018 & 6.332 & 9.228 & 1.580 & 0.00 \\
1.5 & 2.215 & $(5)$ & 96844 & 7.999 & 6.378 & 9.147 & 1.525 & 0.00 \\
1.0 & 6.853 & $(5)$ & 78425 & 7.941 & 6.437 & 9.080 & 1.230 & 0.00 \\
0.5 & 1.512 & $(6)$ & 61875 & 7.880 & 6.475 & 9.036 & 0.809 & 0.00 \\
0.0 & 2.939 & $(6)$ & 47968 & 7.826 & 6.498 & 9.007 & 0.359 & 0.00 \\
-0.5 & 5.796 & $(6)$ & 36831 & 7.760 & 6.513 & 8.987 & -0.099 & 0.00 \\
-1.0 & 1.306 & $(7)$ & 28113 & 7.678 & 6.526 & 8.971 & -0.748 & 0.00 \\
-1.5 & 4.411 & $(7)$ & 21378 & 7.540 & 6.536 & 8.959 & -1.835 & 0.00 \\
-2.0 & 1.682 & $(8)$ & 16205 & 7.344 & 6.544 & 8.950 & -3.484 & 0.00 \\
-2.5 & 4.662 & $(8)$ & 12248 & 7.125 & 6.550 & 8.943 & -5.447 & 0.00 \\
-3.0 & 1.052 & $(9)$ & 9238 & 6.902 & 6.553 & 8.938 & - & 0.00 \\
-3.5 & 2.168 & $(9)$ & 6961 & 6.678 & 6.556 & 8.934 & - & 0.00 \\
-3.8 & 3.358 & $(9)$ & 5880 & 6.534 & 6.556 & 8.930 & - & 0.00 \\
-3.9 & 4.381 & $(9)$ & 5560 & 6.463 & 6.557 & 8.929 & - & 0.19 \\
-4.0 & 5.641 & $(9)$ & 5253 & 6.383 & 6.557 & 8.928 & - & 0.47 \\
-4.1 & 6.724 & $(9)$ & 4962 & 6.311 & 6.557 & 8.928 & - & 0.67 \\
-4.2 & 7.708 & $(9)$ & 4687 & 6.244 & 6.558 & 8.927 & - & 0.80 \\
-4.3 & 8.634 & $(9)$ & 4427 & 6.179 & 6.558 & 8.927 & - & 0.88 \\
-4.4 & 9.492 & $(9)$ & 4179 & 6.117 & 6.558 & 8.927 & - & 0.94 \\
-4.6 & 1.116 & $(10)$ & 3727 & 5.995 & 6.558 & 8.927 & - & 0.98 \\
-4.8 & 1.278 & $(10)$ & 3322 & 5.876 & 6.558 & 8.926 & - & 1.00 \\
-4.9 & 1.360 & $(10)$ & 3136 & 5.816 & 6.558 & 8.926 & - & 1.00 \\
\hline \hline
\end{tabular}

mass and high-mass models. Finally, we need to include convective mixing in our envelopes, so that we may investigate the effect of changing surface composition on evolutionary timescale. These new sequences will be used in our ongoing studies of the white dwarf luminosity function (see Wood 1992).

Acknowledgments: It is a pleasure to thank to Paul Bradley for numerous discussions and Steve Kawaler for starting models evolved from the ZAMS. This work was supported in part by NSF grant AST92-17988 and the 19921993 Earnest F. Fullam Award of the Dudley Observatory.

\section{References}

Beaudet, G., Petrosian, V. and Salpeter, E. E. 1967, ApJ, 150, 979

Canuto, V. M., and Mazzitelli, I. 1991, ApJ, 370, 295

Canuto, V. M., and Mazzitelli, I. 1991, ApJ, 389, 724 
Table 42.3. Evolutionary summary of a $Z=0.0000 .8 M_{\odot}$ model sequence

\begin{tabular}{ccccccccc}
\hline \hline $\log \left(L / L_{\odot}\right)$ & \multicolumn{2}{c}{ Age $(\mathrm{yr})$} & \multicolumn{1}{c}{$T_{\text {eff }}$} & $\log T_{c}$ & $\log \left(\rho_{c}\right)$ & $\log (R)$ & $\log \left(L_{\nu} / L_{\odot}\right)$ & $q_{\text {xtal }}$ \\
\hline 3.0 & 4.927 & $(3)$ & 195469 & 8.248 & 6.646 & 9.287 & 3.049 & 0.00 \\
2.5 & 4.327 & $(4)$ & 172013 & 8.171 & 6.752 & 9.148 & 2.910 & 0.00 \\
2.0 & 1.496 & $(5)$ & 147600 & 8.054 & 6.875 & 9.031 & 2.438 & 0.00 \\
1.5 & 3.039 & $(5)$ & 119074 & 7.982 & 6.928 & 8.968 & 1.949 & 0.00 \\
1.0 & 5.433 & $(5)$ & 93185 & 7.928 & 6.954 & 8.930 & 1.481 & 0.00 \\
0.5 & 9.350 & $(5)$ & 71822 & 7.884 & 6.970 & 8.907 & 1.044 & 0.00 \\
0.0 & 1.656 & $(6)$ & 54885 & 7.843 & 6.980 & 8.890 & 0.596 & 0.00 \\
-0.5 & 3.401 & $(6)$ & 41731 & 7.791 & 6.988 & 8.878 & 0.130 & 0.00 \\
-1.0 & 1.037 & $(7)$ & 31651 & 7.691 & 6.996 & 8.868 & -0.756 & 0.00 \\
-1.5 & 6.440 & $(7)$ & 23975 & 7.495 & 7.005 & 8.860 & -2.410 & 0.00 \\
-2.0 & 2.328 & $(8)$ & 18110 & 7.270 & 7.012 & 8.853 & -4.475 & 0.00 \\
-2.5 & 5.637 & $(8)$ & 13649 & 7.047 & 7.016 & 8.849 & -6.234 & 0.00 \\
-3.0 & 1.213 & $(9)$ & 10271 & 6.822 & 7.018 & 8.846 & - & 0.00 \\
-3.4 & 2.178 & $(9)$ & 8177 & 6.652 & 7.019 & 8.844 & - & 0.07 \\
-3.5 & 2.627 & $(9)$ & 7724 & 6.605 & 7.019 & 8.843 & - & 0.21 \\
-3.6 & 3.148 & $(9)$ & 7295 & 6.562 & 7.019 & 8.843 & - & 0.35 \\
-3.7 & 3.698 & $(9)$ & 6891 & 6.519 & 7.020 & 8.842 & - & 0.48 \\
-3.8 & 4.301 & $(9)$ & 6510 & 6.477 & 7.020 & 8.842 & - & 0.60 \\
-3.9 & 4.970 & $(9)$ & 6151 & 6.434 & 7.020 & 8.841 & - & 0.70 \\
-4.0 & 5.969 & $(9)$ & 5813 & 6.367 & 7.020 & 8.840 & - & 0.81 \\
-4.1 & 7.178 & $(9)$ & 5492 & 6.279 & 7.020 & 8.840 & - & 0.91 \\
-4.2 & 8.165 & $(9)$ & 5187 & 6.199 & 7.021 & 8.839 & - & 0.96 \\
-4.4 & 9.766 & $(9)$ & 4626 & 6.049 & 7.021 & 8.839 & - & 0.99 \\
-4.6 & 1.108 & $(10)$ & 4124 & 5.909 & 7.021 & 8.838 & - & 1.00 \\
-4.7 & 1.166 & $(10)$ & 3894 & 5.839 & 7.021 & 8.838 & - & 1.00 \\
\hline \hline
\end{tabular}

Cox, A. N., and Stewart, J. N. 1970, ApJS, 19, 261

DeZot,ti, G. 1972, Estratto dalle Memorie della Società Astronomica Italiana, 43, 89

Iglesias, C. A., and Rogers, F. J. 1993, ApJ, 412, 752

Itoh, N., Mutoh, H., Hikita, A., and Kohyama, Y. 1992, ApJ, 395, 622

Itoh, N., Hayashi, H., and Kohyama, Y. 1993, ApJ, 418, 405

Itoh, N., and Kohyama, Y. 1993, ApJ, 404, 268 1983, ApJ, 273, 774

Lamb, D. Q. 1974, Ph.D. thesis, University of Rochester

Lamb, D. Q., and Van Horn, H. M. 1975, ApJ, 200, 306

Lenzuni, P., Chernoff, D. F., and Salpeter, E. E. 1991, ApJS, 76, 759

Mazzitelli, I., 1994, these proceedings

Rogers, F. J., and Iglesias, C. A. 1992, ApJS, 79, 507

Wood, M. A. 1990, PhD thesis, The University of Texas at Austin

Wood, M. A. 1992, Ap., 386, 539 